$\mathrm{C}-\mathrm{A} / \mathrm{AP} / \# 414$ Dec 2010

\title{
Creating Intense Polarized Electron Beam via Laser Stripping and Spin-Orbit Interaction
}

\author{
V. Danilov, T. Gorlov, V. Ptitsyn
}

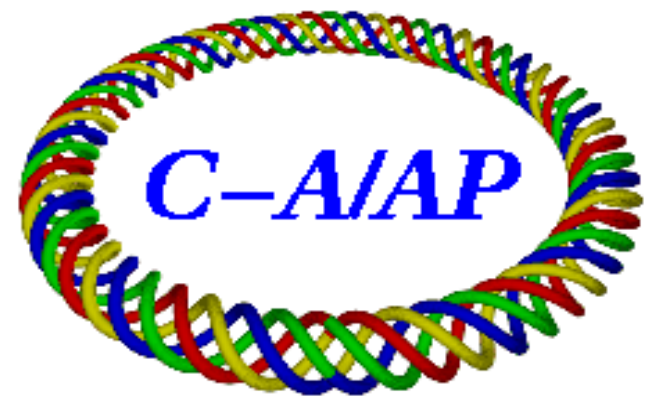

\section{Collider-Accelerator Department Brookhaven National Laboratory Upton, NY 11973}

Notice: This document has been authorized by employees of Brookhaven Science Associates, LLC under Contract No. DE-AC02-98CH10886 with the U.S. Department of Energy. The United States Government retains a non-exclusive, paid-up, irrevocable, world-wide license to publish or reproduce the published form of this document, or allow others to do so, for United States Government purposes. 


\title{
CREATING INTENSE POLARIZED ELECTRON BEAM VIA LASER STRIPPING AND SPIN-ORBIT INTERACTION
}

\author{
V. Danilov, T. Gorlov \\ Spallation Neutron Source Project, Oak Ridge National Laboratory, \\ Oak Ridge, TN 37830 \\ V. Ptitsyn \\ Collider-Accelerator Department, Brookhaven National Laboratory, Upton, NY 11973
}

\begin{abstract}
The recent advance in laser field make it possible to excite and strip electrons with definite spin from hydrogen atoms. The sources of hydrogen atoms with orders of magnitude higher currents (than that of the conventional polarized electron cathods) can be obtained from $\mathrm{H}^{-}$sources with good monochromatization. With one electron of $\mathrm{H}^{-}$stripped by a laser, the remained electron is excited to upper state $\left(2 \mathrm{P}^{3 / 2}\right.$ and $\left.2 \mathrm{P}^{1 / 2}\right)$ by a circular polarization laser light from FEL. Then, it is excited to a high quantum number $(n=7)$ with mostly one spin direction due to energy level split of the states with a definite direction of spin and angular momentum in an applied magnetic field and then it is stripped by a strong electric field of an RF cavity. This paper presents combination of lasers and fields to get high polarization and high current electron source.
\end{abstract}

\section{Introduction}

A polarized electron source, producing average current of tens $\mathrm{mA}$, is required for ERLbased electron-ion collider eRHIC [1], which considerably exceeds the average current produced by present "state-of-the-art" electron sources using the photoemission from strained GaAs cathode. Recently, high efficiently methods of laser-assisted electron stripping of hydrogen were developed (see, e.g. [2-4]) and experimentally demonstrated in Oak Ridge [5]. This was done with around $1 \mathrm{GeV}$ SNS beam. Using the same beam for electron sources proposed in this paper is not practical because the cost of the project. Therefore, to minimize the source cost, the energy of the carrier ions has to be as low as possible. At the same time the ion energy has to be sufficient to prevent space charge effects and add some Doppler effect to do the selective excitation of the states with different spin. The initial current source has to be $\mathrm{H}^{-} \mathrm{CW}$ source that has to produce 50 $\mathrm{mA}$ current (sources with $20 \mathrm{~mA}$ are available, but the upgrade with higher current and larger beam emittance is possible [6]). The initial acceleration to a few hundred $\mathrm{keV}$ has to be done either by low frequency RFQ or some other accelerator to match the $14 \mathrm{MHz}$ frequency of the proton beam in RHIC. Then, the $\mathrm{H}^{-}$beam goes through the sequence of dipoles and quads creating a correlation of energy and angles and reducing its angular spread to minimize the absorption line width of the hydrogen states [3]. In addition, we have to reduce the ion bunch length in the dispersion section in order to make the electron beam, produced by the source, appropriate for further compression. After this, the first electron is easily detached by $1064 \mathrm{~nm}$ laser. On the next step the neutral hydrogen is excited by circularly polarized around $100 \mathrm{~nm}$ light from the FEL (the linac of the FEL can be the ERL used for electron pre-acceleration for eRHIC). The electrons end up in $2 \mathrm{P}^{3 / 2}$ and $2 \mathrm{P}^{1 / 2}$ states. Immediately after this the states are laser-excited to states with high 
principal quantum number $\mathrm{n}$ ( $\mathrm{n}$ around 7). This process must happen in longitudinal magnetic field where the upper state level energy depends only on value of spin doubled plus angular momentum of the electron (lower $n=2$ state less affected by the field due to strong spin-orbital interaction). We excite $n=7, l=2, m=2,1$ states with longitudinal spin along the field. Then, $\mathrm{H}$ atoms at this long-living state enter an RF cavity with high field where the electron is stripped and accelerated thus producing polarized electrons. The remaining proton beam could be recycled and used for other applications if needed. Figure 1 shows all the described steps.

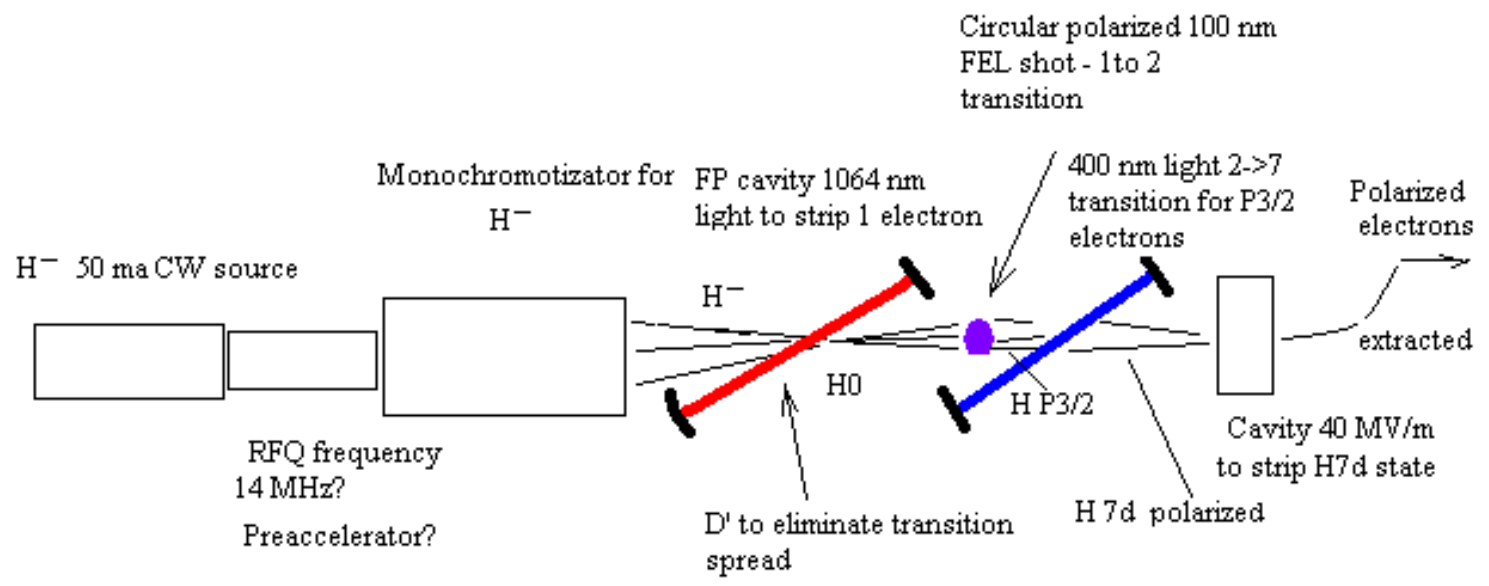

Figure 1 Outline of the source.

In the next section, we describe the parameters of the lasers and the ion beam.

\section{Ion and Laser Beam Parameters}

\section{Initial acceleration}

We have to deal with dilemma at this stage: it is either to stick with typical RFQ with frequencies of hundredth of $\mathrm{MHz}$ and make longitudinal manipulation with electron bunches later or develop $14 \mathrm{MHz}$ accelerator to get bunches with the right $14 \mathrm{MHz}$ 
frequency for eRHIC. We have it as an open question but for the beam parameters we will use SNS $40 \mathrm{~mA}$ linac beam. It has $100 \mathrm{ps}$ bunch length, $2.5 \mathrm{MeV}$ energy and $20 \mathrm{keV}$ energy spread with transverse normalized emittance around $0.3 \mathrm{~mm} * \mathrm{mrad}$. Of course, the energy of the beam will be lower for our purpose, but we think the parameters won't deviate more than factor 3 from those of SNS ones. That is enough for our estimations below.

\section{Monochromatization}

The initial acceleration is needed to bunch the beam, to prevent space charge effects, and to create smaller spread of transition frequencies from $2^{\text {nd }}$ to $7^{\text {th }}$ levels. For example, the relative spread of transition frequencies in the source is of the order of $10^{-3}$, but after RFQ acceleration (SNS case) it is an order of magnitude lower (about $2 \cdot 10^{-4}$ ). It is still requires large laser power for 2 to 7 excitation and transition frequency spread has to be smaller than the split of level with opposite spin orientation, which is of the order of $1.5 \cdot 10^{-5}$. Therefore, we need more than order of magnitude reduction of the transition frequencies spread to get low power final stage laser and good separation of transition frequency to selectively excite states with needed polarization. To achieve this we employ the trick from [3], namely, we introduce the dispersion function in the stripping region such that the angles are correlated with energy and the derivative of the Doppler formula for laser light Lorentz transformation on energy is equal to zero. For this, the dispersion derivative $D^{\prime}$ must be:

$$
D^{\prime}=-\frac{\beta+\cos \alpha}{\sin \alpha},
$$

where $\beta$ is relativistic factor and $\alpha$ is the incident angle between the laser light and the ion beam axis. The optics after initial acceleration has to include magnets for the dispersion creation. Practical values of $D^{\prime}$ have to be of the order of 1 . Also we need large horizontal beta-function to reduce the angular spread of the beam which is a straightforward step.

\section{First electron stripping}

The first electron stripping is the easiest part - it is routinely done at SNS and other labs for diagnostic purposes. Here we need to strip the entire beam so the system requires a bit more power than the typical diagnostics setup. The estimation for the laser power in head-on collision is the following:

$$
P_{a}=5 \frac{\sigma_{\text {beam }} E_{\text {photon }}}{\sigma_{\text {det }}} \frac{f_{\text {rep }}}{Q_{\text {cavity }}} \approx 2.4 \mathrm{~W},
$$

where photo detachment cross section $\sigma_{\text {det }} \approx 3.510^{-17} \mathrm{~cm}^{2}$ for low energy ion beam and $1064 \mathrm{~nm}$ light in head-on collision, $E_{\text {photon }} \approx 1.2 \mathrm{eV}, \sigma_{\text {beam }} \approx 0.01 \mathrm{~cm}^{2}, f_{\text {rep }}=14 \mathrm{MHz}$ is the repletion rate of the ion and laser pulses, is the percentage of time the system is in operation, Q cavity $=1000$ is the practically achievable amplification of Fabri-Perot cavity in infrared region, and total factor 5 in front of the expression is the number of 
exponential decay lengths needed to get more than $99 \%$ of stripping. One can see that the estimation for the average laser power yields a small number. The energy change of 1.2 $\mathrm{eV}$ is negligible as compared to the beam energy spread.

\section{Excitation to the $2^{\text {nd }}$ level}

This is by far the most expensive part of the device, since a FEL is needed to produce UV light. In eRHIC, we can get the advantage of using eRHIC components to drastically reduce the price tag. The electron beam, to drive the FEL, can be produced by an SRF electron gun, similar to $703 \mathrm{MHz}$ SRF gun being developed at BNL for its ERL test facility [8]. Such electron gun is also considered for providing an electron beam for eRHIC-based X-ray FELs [9]. The acceleration of the electrons to the energy of several hundred $\mathrm{MeV}$ can be done in a pre-accelerator ERL used in the eRHIC design to accelerate the eRHIC polarized electron beam up to $0.6 \mathrm{GeV}$ energy. The acceleration of the eRHIC and FEL electron beams goes simultaneously, both beams having $14 \mathrm{MHz}$ bunch structure. The beams have to be accelerated in different RF phases so they can be effectively separated in a magnet separator after the ERL due to the energy difference.

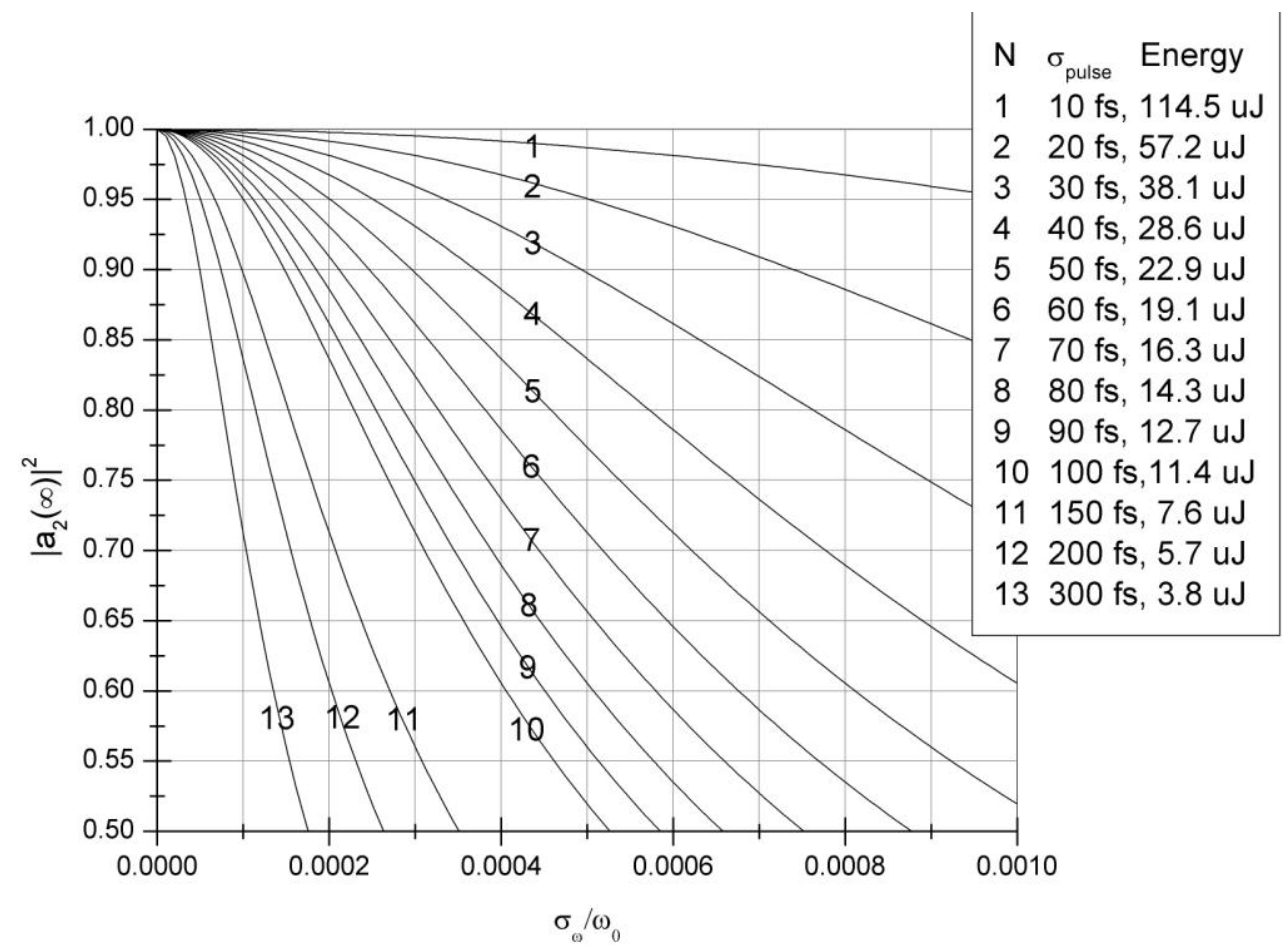

Figure 2 Excitation probability of $n=2$ level by a short FEL pulse.

In order to reach $100 \mathrm{~nm}$ frequency of FEL lasing the high-gain harmonic generation FEL scheme [7] can be utilized. Two additional undulators (a modulator and a radiator) are required to produce high harmonic narrow bandwidth $100 \mathrm{~nm}$ pulses with frequency 14 MHz. After passing the lasing sections the beam is directed to the pre-accelerator ERL for the energy recovery. The quantum process of head-on excitation is shown in Figure 2. 
It shows excitation probability versus relative transition frequency offset, related to the Doppler effect. All solid curves show the whole beam excitation probability for the fixed FEL pulse width - the total energy of the pulse, multiplied by this width is constant (and equal to $1.14 \cdot 10^{-18} \mathrm{~J} \cdot \mathrm{s}$ ) in these calculations. Figure 3 shows the process of excitation for particular particles with most representative energy offsets.

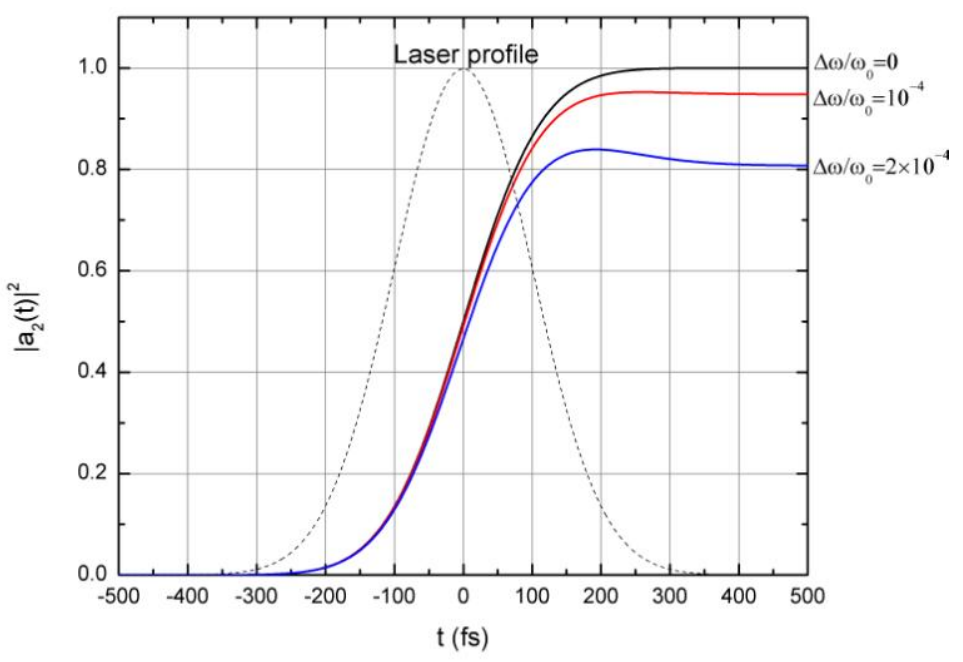

Figure 3 Probability of excitation for Gaussian $100 \mathrm{fs}$ rms pulse for zero (black), $10^{-4}$ (red), and $2 \cdot 10^{-4}$ (blue) relative transition frequencies offsets.

The excitation is done here by a Gaussian laser pulse of 100 fs r.m.s. with radial r.m.s 1 $\mathrm{mm}$ with total light pulse energy of $12 \mu \mathrm{J}$ that is well within obtainable $100 \mu \mathrm{J}$ at BNL NSLS FEL [7]. The short pulse is needed to cover the spectrum of transition frequencies for hydrogen in head-on collision where dispersion monochromatization doesn't work. The quality of light (spectrum width) has to be not worse than .1\%. In addition, we have to mention that the light has to have a circular polarization to excite fewer levels thus the electric laser field has to have a factor $e^{i \varphi}$, where $\varphi$ is the angle around beam axis. The estimate for total excitations is around $95 \%$.

\section{Excitation to $n=7$ state}

After the electron in $\mathrm{n}=2$ state is excited, it is uplifted by $400 \mathrm{~nm}$ blue laser (second harmonic of Ti:Saphire laser) to $n=7$ state. The transition happens to $l=2$ state if again the light has the same as FEL circular polarization. The physics of excitation is the most delicate piece of the proposal and needs to be described in more details.

First of all, we have to explain the choice of longitudinal magnetic field. If it is absent, the electron is mostly unpolarized in $n=2$ state and its transition into $n=7$ state leaves it unpolarized. If the field is too strong the states with spin up and down along the field are eigenstates for both $\mathrm{n}=2$ and $\mathrm{n}=7$ states. Therefore, the initial unpolarized electron can have transitions only to states with the same spin, leaving us with no polarization. In the medium field ( $\mathrm{B}=0.78 \mathrm{~T}$ in our case) the $\mathrm{n}=2$ state is less affected and its states with 
different total momentum and its projections have both up and down spin components. The upper states with $n=7$ in this field have only definite direction of spin along the field. If we take two upper states with $1=2, m=2, m=1$, the spin will be up, and there will be nonzero transitions to these two states from all three excited $n=2$ state, so eventually, after tuning 3 laser beams, we excite electron only to spin up state (with some small transitions to spin down $\mathrm{m}=2$ state that reduces the polarization slightly). Now we present wave functions, dipole transition coefficients, and energies of the states. The eigenfunctions $u$ of the $\mathrm{n}=2$ states are (see [10], p. 212):

$$
\begin{aligned}
& u=R_{n l}(r)\left(\begin{array}{l}
\sqrt{\frac{1+\delta}{2}} Y_{l, m-1 / 2} \\
-\sqrt{\frac{1-\delta}{2}} Y_{l, m+1 / 2}
\end{array}\right) \text { for the higer level, } \\
& u=R_{n l}(r)\left(\begin{array}{l}
\sqrt{\frac{1-\delta}{2}} Y_{l, m-1 / 2} \\
\sqrt{\frac{1+\delta}{2}} Y_{l, m+1 / 2}
\end{array}\right) \text { for the lower level, }
\end{aligned}
$$

where $R$ and $Y$ are conventional radial and angular functions, with $n=2, l=1, \mathrm{~m}=3 / 2,1 / 2$ for the higher $\mathrm{P}^{3 / 2}$ level, and $\mathrm{m}=1 / 2$ for the lower $\mathrm{P}^{1 / 2}$ level (the only ones excited for our case with circular polarization excitation), $\delta=\frac{\xi+\frac{2 m}{2 l+1}}{\sqrt{1+\xi \frac{4 m}{2 l+1}+\xi^{2}}}, \xi=B \mu_{0} / \Delta E$, where $\mu_{0}$ is Bohr's magneton, $B$ is the magnetic field, and $\Delta E$ is spin-orbital energy split for the level. We take, for example, $\xi=1$ that corresponds to $B=0.78$ Tesla. For $\mathrm{n}=7$ level $\delta$ is very close to 1 , so we put it just equal to 1 . The wave functions of interest for this level are:

$$
\begin{aligned}
& u=R_{72}(r) Y_{2, m}\left(\begin{array}{l}
1 \\
0
\end{array}\right) \text { for the } m=1,2 \text { levels spin up, } \\
& u=R_{72}(r) Y_{2, m}\left(\begin{array}{l}
0 \\
1
\end{array}\right) \text { for } m=2 \text { level spin down, }
\end{aligned}
$$

where $\mathrm{m}$ here stands only for angular momentum projection onto longitudinal axis.

The energies are the following: the unperturbed energy level value is $3.122 \mathrm{eV}$ between $n=2 P^{3 / 2}$ and $n=7$ state. We will keep it in mind that a laser around $400 \mathrm{~nm}$ wavelength is needed for excitation, but we calculate all other energy differences with subtraction of this number for brevity. Thus, the levels $n=7, m=2$ spin up and $n=2 P^{3 / 2}$ $\mathrm{m}=3 / 2$ have approximately energy difference of $4.5 \cdot 10^{-5} \mathrm{eV}$ in our magnetic field. This difference is $610^{-5} \mathrm{eV}$ for $\mathrm{n}=7, \mathrm{~m}=2$ spin up and $\mathrm{n}=2 \mathrm{P}^{3 / 2} \mathrm{~m}=1 / 2$ levels, and $12 \cdot 10^{-5} \mathrm{eV}$ for and $\mathrm{n}=7, \mathrm{~m}=2$ spin up and $\mathrm{n}=2 \mathrm{P}^{1 / 2} \mathrm{~m}=1 / 2$ levels. All these transitions work in our favor 
and their dipole transition coefficients (the longitudinal axis is $\mathrm{z}$ axis in our case and the light has circular polarization as FEL light, i.e. its electric field is proportional to $\left.e^{i \varphi}\right) \mu_{n k}=-\int d^{3} r u_{k}^{*}(\vec{r}) e r \sin \theta e^{i \varphi} u_{n}(\vec{r})$ (k is related to the upper and $\mathrm{n}$ to the lower state, respectively) are: $0.44,0.296,0.094$ in units of $e a_{0}$, where $a_{0}$ is the Bohr's radius. For the first two transitions the coefficients are large and the needed power is low. In the manner of paper [2] one can obtain probabilities of excitation. Figure 3 shows the excitation of electrons with 1, 2, 3 r.m.s. of transition frequencies for the first transition (we believe we can get the transition frequency r.m.s. equal to $0.5 \cdot 10^{-5}$ in relative units that is three times smaller than the relative spin-orbital split for $n=2$ state).

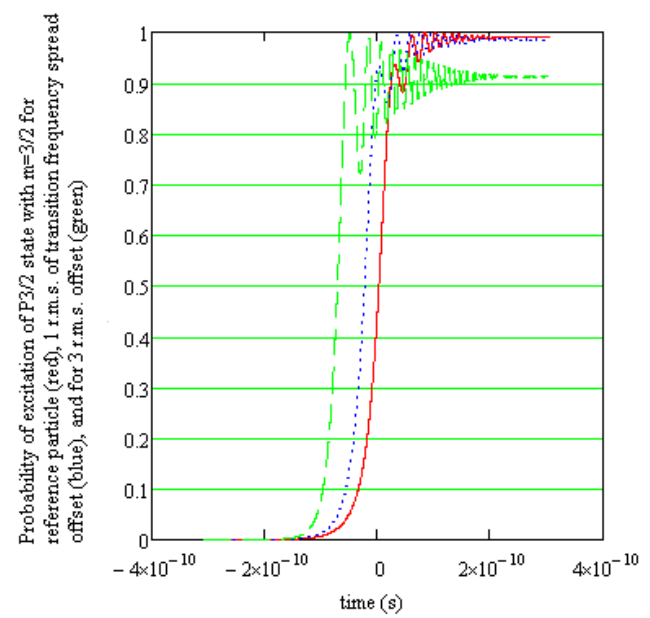

Figure 4 Probabilities for $\mathrm{P}^{3 / 2} \mathrm{~m}=3 / 2$ state to $\mathrm{n}=7,1=2, \mathrm{~m}=2$ spin up state excitation.

The laser peak power is $50 \mathrm{~kW}$ in 100 ps r.m.s. pulses with $1 \mathrm{~mm}$ r.m.s transverse size vertical size and $1 \mathrm{~cm}$ horizontal size with frequency $14 \mathrm{MHz}$. The average power is 75 $\mathrm{W}$ that is a reasonable number. The second transition with slightly less dipole transition is almost the same (needs roughly 2 times more laser power). One can see that the excitation above $95 \%$ is easily achievable (we have to mention that we employ also divergence of the beam for excitation (see [2]) and the change of laser frequency on r.m.s. beam size is $2 \cdot 10^{-5}$ ). The transverse size is chosen to have time for particle to get excited with good separation (if it is too small the particles will traverse the beam fast and the laser light spectrum for them will be very broad to separately excite states) with their relativistic beta around 0.015 (we choose this beta for being enough to manipulate with particles and at the same time to keep the bunching and acceleration of ions inexpensive). The last useful transition requires more energy (about 10 times) since its dipole transition coefficient is lower. The probabilities of excitation for the same laser beam but 20 times more power look like ones from Figure 3. The last piece of the process is parasitic transitions which excites state with the opposite spin. The strongest one is $\mathrm{P}^{1 / 2} \mathrm{~m}=1 / 2$ to $\mathrm{n}=7,1=2, \mathrm{~m}=2$ spin down. It has 3 r.m.s. frequency offset from $\mathrm{P}^{1 / 2} \mathrm{~m}=1 / 2$ to $\mathrm{n}=7, \mathrm{l}=2$, 
$\mathrm{m}=1$ spin up transition and the dipole transition coefficient is rather large, being equal to 0.419 . Here we reduce frequency change parameter by half to reduce clipping of these state by an edge of power spectrum. Figure 4 shows that it is excited slightly by the same light that is used for $\mathrm{n}=7, \mathrm{~m}=2$ spin up and $\mathrm{n}=2 \mathrm{P}^{1 / 2} \mathrm{~m}=1 / 2$ transition.

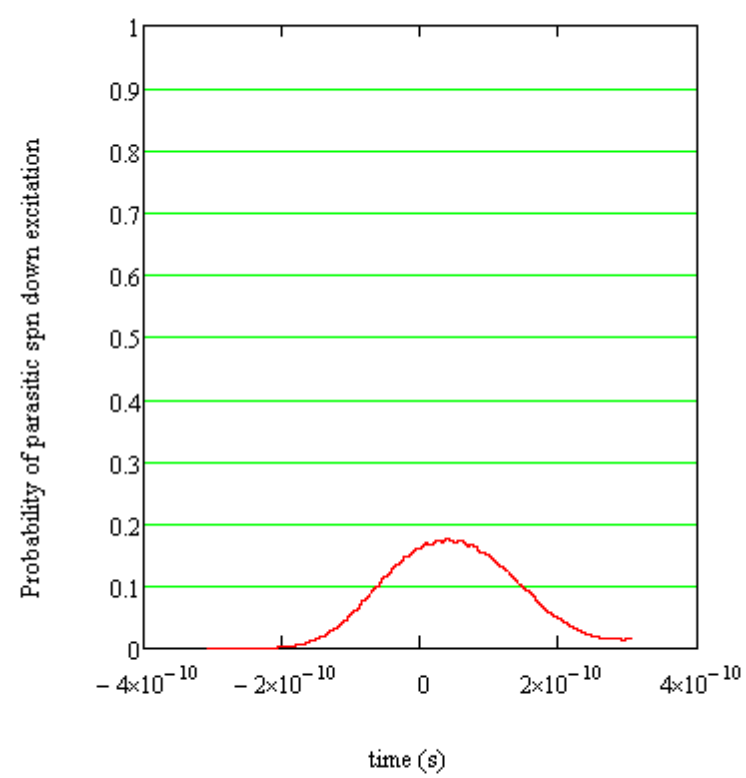

Figure 5 Probability of parasitic excitation.

In reality, both transitions have to be simulated simultaneously - it will be done later. We expect this parasitic level is to be excited not more than $10 \%$ percent thus the total polarization from 4 transitions (assuming first $3 \mathrm{n}=2$ levels are roughly equally distributed) is not less than $90 \%$.

The laser, used for the last useful transitions should have around $1500 \mathrm{~W}$ average power. To reduce it, a Fabri-Perot cavity could be employed. With $\mathrm{Q}=10$ for blue light the average laser power drops to $150 \mathrm{~W}$ which is a reasonable number. All three beams, including the Fabri-Perot cavity excitation beam could be obtained from one laser through the system of mirrors and splitters.

\section{Stripping electrons from $n=7$ state}

For electron stripping and acceleration we need a reasonable RF cavity (probably, a superconducting one). The available fields are in the range of $40 \mathrm{MV} / \mathrm{m}$. Figures 5 and 6 show the lifetime of the levels for $n=6$, and $n=7$, respectively. We need the lifetime to be less than picoseconds to prevent elongating of the bunch. One can see that for $n=7$ 
$40 \mathrm{MV} / \mathrm{m}$ field reduces the lifetime to this level. This determines the choice of the upper state. The wavelength of the cavity is determined by the length of the bunch. We expect it to be around $1 \mathrm{~ns}$, as in conventional case. Then, the variation of the electric field has to be less than $10 \%$ to strip the last electron - it results in $70 \mathrm{MHz}$ cavity.

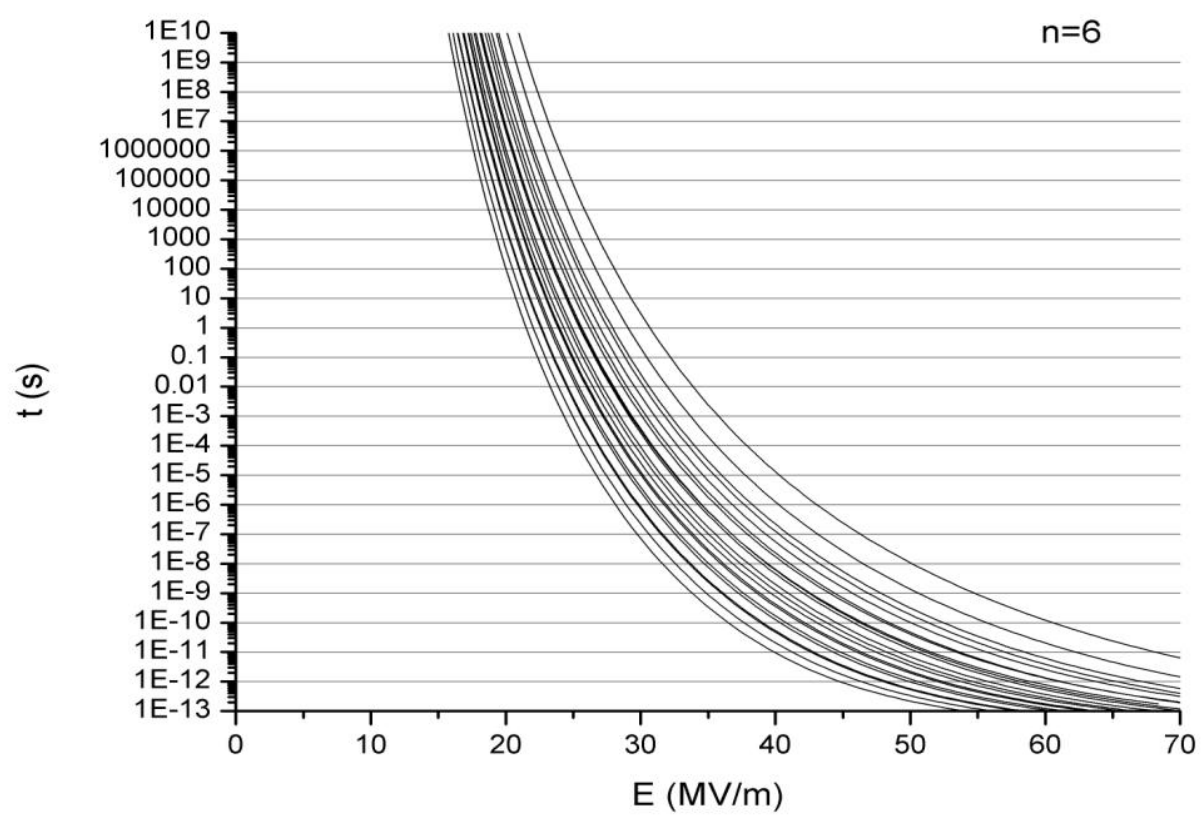

Figure 6 Lifetime of $n=6$ as a function of electric field. 


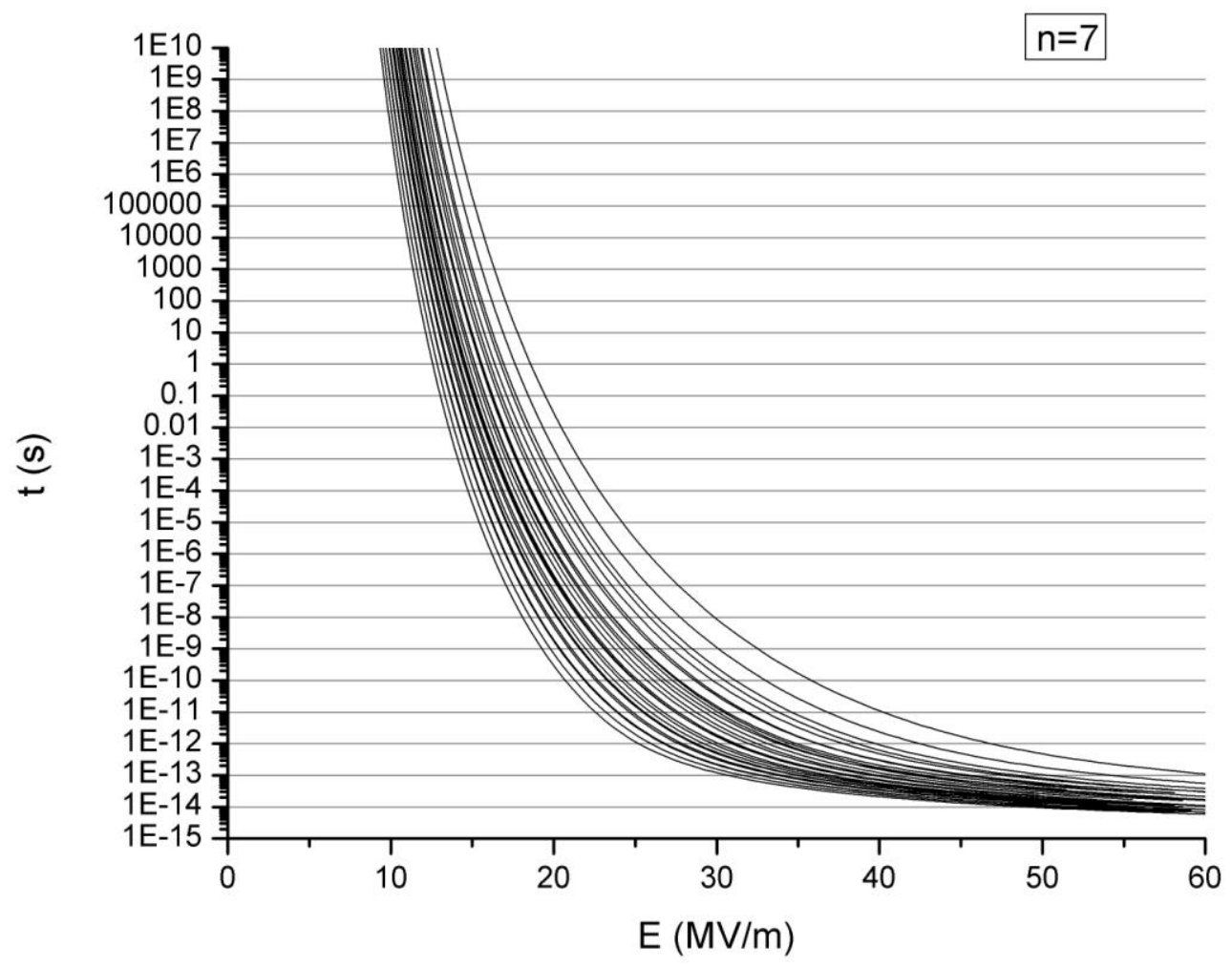

Figure 7 Lifetime of $\mathbf{n}=7$ as a function of electric field.

\section{Bunch compression}

The electron beam after the cavity will have about the same duration as the ion beam (few hundred picoseconds) and the energy, acquired in the cavity is expected to be around $10 \mathrm{MeV}$. Therefore, the longitudinal parameters of the electron beam are comparable with those of the conventional RF cathode beams and the compressor parameters have to be similar to the standard ones.

The transverse emittance of the electron beam.

The electron beam inherits some parameters of the ion beam, for example, its transverse size that we take to be around $1 \mathrm{~mm}$. As for the angles, its more complicated question. If it inherits the angles also in the RF cavity, then its transverse emittance will be order of magnitude smaller than that of the conventional cathodes (some fraction of $1 \mathrm{~mm} \cdot \mathrm{mrad}$ ). But the Stark effect stripping may introduce some angular spread also. This has to be estimated later.

\section{Acknowledgements}

This research is supported by UT-Battelle, LLC and by FRA, LLC for the U. S. Department of Energy under contracts No. DE-AC05-00OR22725 and No. DE-AC0298CH10886. 


\section{REFERENCES}

1. V. Ptitsyn, "eRHIC machine design status", EIC Collaboration meeting, Washington DC, (2010)

http://faculty.cua.edu/hornt/EICC_CUA_2010/Fri30July/Ptitsyn_eRHIC_Design. pptx

2. I. Yamane, ICFA-HB2002, AIP Conference Proc. 642, Batavia, Il (2002) p.335.

3. V. Danilov et al, "Three-step H- charge exchange injection with a narrow-band laser", Phys. Rev. ST Accel. Beams 6, 5 (2003) 053501.

4. T. Gorlov et al, Phys. Rev. ST Accel. Beams 13, 5 (2010) 050101.

5. V. Danilov et al, "Proof-of-principle demonstration of high efficiency laserassisted $\mathrm{H}^{-}$beam conversion to protons", Phys. Rev. ST Accel. Beams 10, 5 (2007) 053501 .

6. V. Dudnikov, private communication.

7. L.H. Yu, et. al., "First Ultraviolet High-Gain Harmonic-generation Free-Electron laser", Phys. Rev. Letters, v 91, 7, August (2003) 074801.

8. V.N. Litvinenko, et al., "R\&D Energy Recovery Linac at Brookhaven National Laboratory", MOPC057, EPAC'08, Genoa, (2008).

9. V.N. Litvinenko, et al., "FEL Potential of eRHIC", TUPA22, 32 ${ }^{\text {nd }}$ FEL Conference, Malme (2010).

10. H. A. Bethe and E. Salpeter, "Quantum mechanics of One- and Two-Electron Atoms", Springer-Verlag, New York (1957). 